\title{
Automatic Role Recognition in Multiparty Recordings: Using Social Affiliation Networks for Feature Extraction
}

\author{
Hugues Salamin, Sarah Favre and Alessandro Vinciarelli
}

\begin{abstract}
Automatic analysis of social interactions attracts increasing attention in the multimedia community. This paper considers one of the most important aspects of the problem, namely the roles played by individuals interacting in different settings. In particular, this work proposes an automatic approach for the recognition of roles in both production environment contexts (e.g., news and talk-shows) and spontaneous situations (e.g., meetings). The experiments are performed over roughly 90 hours of material (one of the largest databases used for role recognition in the literature) and show that the recognition effectiveness depends on how much the roles influence the behavior of people. Furthermore, this work proposes the first approach for modeling mutual dependences between roles and assesses its effect on role recognition performance.
\end{abstract}

Index Terms-Role Recognition, Social Network Analysis, Broadcast Data, Meeting Recordings.

\section{INTRODUCTION}

The computing community is making significant efforts towards the development of automatic approaches for the analysis of social interactions (see [1][2][3] for extensive surveys of the domain). This is not surprising as social interactions are not only one of the most important aspects of our everyday lives, but also an ubiquitous subject in multimedia data: radio and television programs (debates, news, talk-shows, movies, etc.) rarely show something else than social interactions. The way people interact depends on the context, but there is one aspect that all social interactions seem to have in common:

People do not interact with one another as anonymous beings. They come together in the context of specific environments and with specific purposes. Their interactions involve behaviors associated with defined statuses and particular roles. These statuses and roles help to pattern our social interactions and provide predictability [4].

Copyright (c) 2008 IEEE. Personal use of this material is permitted. However, permission to use this material for any other purposes must be obtained from the IEEE by sending a request to pubs-permissions@ieee.org.

H.Salamin and A.Vinciarelli are with University of Glasgow (Department of Computing Science), Lilybank Gardens, Glasgow, G12 8QQ, Scotland, UK. S.Favre is with Idiap Research Institute, CP592 1920 Martigny (Switzerland), and École Polytechnique Fédérale de Lausanne (EPFL), 1015 Lausanne (Switzerland). When the work was done, H.Salamin and A.Vinciarelli were with Idiap Research Institute. E-mail: sfavre@idiap.ch, \{hugues.salamin, alessandro.vinciarelli\}@dcs.gla.ac.uk. This work is supported by the Swiss National Science Foundation (under the National Centre of Competence in Research on Interactive Multimodal Information Management), and by the European Community's Seventh Framework Programme (FP7/2007-2013), under grant agreement no. 231287 (SSPNet).
As the above suggests that roles are a universal key to understand social interactions and these are one of the most common subjects of multimedia material, this work proposes an approach for the automatic recognition of roles in multiparty recordings.

The approach includes two main stages (see Figure 1): the first is the feature extraction and it involves the automatic construction of a Social Affiliation Network [5] as well as its conversion into feature vectors that represent each person in terms of their relationships with the others. The second stage is the role recognition, i.e. the mapping of the feature vectors extracted in the first stage into roles belonging to a predefined set. This task is performed using Bernoulli or Multinomial distributions [6] for the Affiliation Network features and Gaussian distributions for the intervention lengths associated to each role.

The experiments have been performed over three different corpora (see Section V-A for more details): a collection of radio news bulletins (around 20 hours), a dataset of radio talk-shows (around 25 hours), and the AMI meeting corpus (around 45 hours) [7]. To the best of our knowledge, there is only one work reporting experiments performed over a larger amount of data [8]. However, the corpus of [8] includes only the news scenario, while our data includes other settings. This is important because it allows one to assess the approach robustness with respect to changes of the interaction structure.

For the first two datasets, the accuracy (percentage of recording time correctly labeled in terms of role) ranges from 60 to $85 \%$, for the third dataset the accuracy is around $45 \%$. One possible explanation of the difference is that roles are easier to model when they are formal, i.e. correspond to functions that impose more or less rigorous constraints on the way people behave and interact with the others (like in the case of broadcast data). In contrast, roles are harder to model when they are informal, i.e. when they correspond to a position in a given social system (e.g. manager in a company) and do not necessarily impose tight constraints on the way people behave and interact (like in the case of meetings). However, the performance significantly outperforms chance for both broadcast and meeting recordings.

Role recognition can be useful in several applications (the list is not exhaustive). For example in media browsers, the information about the role of the person speaking at a given moment can help users to quickly identify segments of interest. In summarization, the role of people can be used as a 


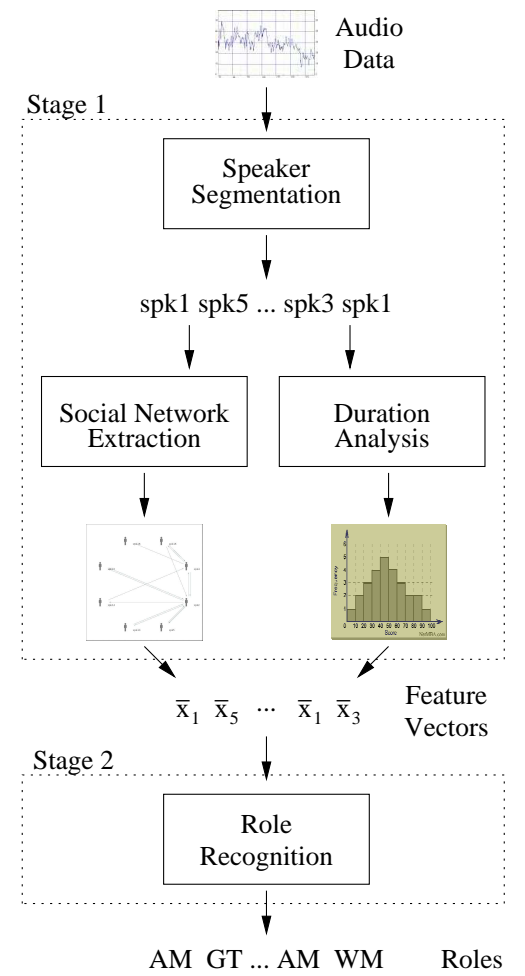

Fig. 1. Role recognition approach. The picture shows the two main stages of the approach: the features extraction and the actual role recognition.

criterion to select representative segments of the data [9][10]. In Information Retrieval, the role can be used as an index to enrich the content description of the data. Furthermore, the role can be used to segment the data into semantically coherent segments [11][12].

The main contributions of this paper with respect to previous approaches proposed by the authors [13] and the rest of the literature are as follows:

- The approach proposed in [13] can be applied only to groups involving at least 8-10 persons because it is based on simple Social Networks and these need at least this number of people to produce meaningful features. This work addresses such a limit by introducing the use of Social Affiliation Networks, a different kind of network that makes it possible to analyze smaller groups. Without this change, the analysis of the AMI meetings (including only four participants) would not be possible.

- The approach in [13] does not take into account the dependence between roles. Each person is assigned a role independently of those assigned to others. This work proposes an approach to overcome this limit and takes into account the constraints that the role distribution across different interacting participants must respect. To the best of our knowledge, this is a novelty not only with respect to [13], but also with respect to the state-of-theart.

- To the best of our knowledge, this is the first work in the literature that reports experiments performed over different interaction contexts, i.e., production environment data involving formal roles (news and talk shows) and spontaneous settings involving informal roles (meetings). The rest of the paper is organized as follows: Section II presents a survey of related works, Section III describes the feature extraction stage, Section IV describes the role recognition stage, Section $\mathrm{V}$ presents experiments and results, and Section VI draws some conclusions.

\section{RELATED WORK}

Role recognition works presented in the literature (see [1][3] for survey) can be split into two major groups depending on whether they address the recognition of formal or informal roles [14]. The former correspond to specific functions to be fulfilled in a given social context (e.g., the chairman in a meeting) and tend to induce stable, machine detectable, behavioral patterns. The latter correspond to positions in a social system (e.g., the manager in a company) and do not necessarily result into detectable behavioral patterns.

Most of the works dedicated to formal roles perform experiments over production environment data like movies, news, talk-shows, etc. Some approaches [8][15] apply techniques like Hidden Markov Models or boosting and use features accounting for the speaking activity of people, e.g. intervention length, number of interventions, lexical choices (distributions of bigrams and trigrams), etc. Other approaches [13][16] have proposed the use of Social Networks as a mean to extract features that are given as input to Bayesian classifiers [13] or used to build co-occurrence matrices aimed at identifying social groups [16].

The recognition of informal roles is typically performed using meeting recordings. The work in [17] recognizes social roles suggested by human sciences (e.g., gate-keeper or attacker) by feeding Support Vector Machines with features extracted from both audio and video. These include the same features described above for formal roles and fidgeting measures extracted from the video. The approaches in [19] and [20] are tested over the same meeting data as those used in this work (see Section V-A). The first work combines a Bayesian classifier fed with features extracted using Social Networks, and boosting techniques applied to the distribution of words, bigrams and trigrams extraced from the automatic transcriptions of the interventions. The second work uses speaking activity features (e.g., probability of initiating a talkspurt when someone else is speaking or when a participant in a specific other role is speaking). The AMI meeting corpus has been used as well for automatic recognition of dominant clique (the two most dominant persons) [21] and relationship between dominance and one of the roles played in the corpus (the Project Manager) [22]. While these two works cannot be said to address specifically the role recognition problem, still are similar to the others presented in this section as they identify persons with specific social characteristics depending on their behavior.

\section{FEATURE EXTRACTION}

This section presents the feature extraction stage aimed at extracting and representing the interaction pattern of each 
TABLE I

SYNOPSIS OF ROLE RECOGNITION RESULTS. THE TABLE PROVIDES A BRIEF DESCRIPTION OF THE DATA USED IN THE LITERATURE, AS WELL AS THE PERFORMANCE ACHIEVED IN THE DIFFERENT WORKS.

\begin{tabular}{|c|l|c|c|l|}
\hline Ref. & Data & Time & Roles & Performance \\
\hline \hline$[8]$ & $\begin{array}{l}\text { TDT4 Mandarin broadcast news (336 } \\
\text { shows, 3 roles) }\end{array}$ & $170 \mathrm{~h} .00 \mathrm{~m}$ & formal & $\begin{array}{l}77.0 \% \text { of the news stories correctly labeled in terms } \\
\text { of role }\end{array}$ \\
\hline$[13]$ & $\begin{array}{l}\text { Radio news bulletins (96 recordings, 6 } \\
\text { roles) }\end{array}$ & $25 \mathrm{~h} .00 \mathrm{~m}$ & formal & $\begin{array}{l}85 \% \text { of the data time correctly labeled in terms of } \\
\text { role }\end{array}$ \\
\hline$[15]$ & $\begin{array}{l}\text { NIST TREC SDR Corpus (35 recordings, } \\
\text { publicly available 3 roles) }\end{array}$ & $17 \mathrm{~h} .00 \mathrm{~m}$ & formal & $\begin{array}{l}80.0 \% \text { of the news stories correctly labeled in terms } \\
\text { of role }\end{array}$ \\
\hline$[16]$ & $\begin{array}{l}\text { Movies and TV shows (10 movies and 3 } \\
\text { TV shows, 9-20 roles) }\end{array}$ & $21 \mathrm{~h} .00 \mathrm{~m}$ & formal & $\begin{array}{l}95 \% \text { of leading roles correctly assigned and 84.3\% } \\
\text { of community roles correctly assigned }\end{array}$ \\
\hline \hline$[17]$ & $\begin{array}{l}\text { The Mission Survival Corpus (11 record- } \\
\text { ings, publicly available, 5 roles) }\end{array}$ & $4 \mathrm{~h} .30 \mathrm{~m}$ & informal & $\begin{array}{l}90 \% \text { of analysis windows (around 10 seconds long) } \\
\text { correctly classified in terms of task area roles and } \\
95 \% \text { in terms of socio area roles }\end{array}$ \\
\hline$[18]$ & Meetings (2 recordings, 3 roles) & $0 \mathrm{~h} .45 \mathrm{~m}$ & informal & $\begin{array}{l}53.0 \% \text { of segments (up to 60 seconds long) correctly } \\
\text { classified }\end{array}$ \\
\hline$[19]$ & $\begin{array}{l}\text { AMI Meeting Corpus (138 recordings, } \\
\text { publicly available, 4 roles) }\end{array}$ & $45 \mathrm{~h} .00 \mathrm{~m}$ & informal & $\begin{array}{l}53 \% \text { of the data time correctly labeled in terms of } \\
\text { role }\end{array}$ \\
\hline$[20]$ & $\begin{array}{l}\text { AMI Meeting Corpus (138 recordings, } \\
\text { publicly available, 4 roles) }\end{array}$ & $45 \mathrm{~h} .00 \mathrm{~m}$ & informal & $\begin{array}{l}67.9 \% \text { of the data time correctly labeled in terms of } \\
\text { role }\end{array}$ \\
\hline
\end{tabular}

events

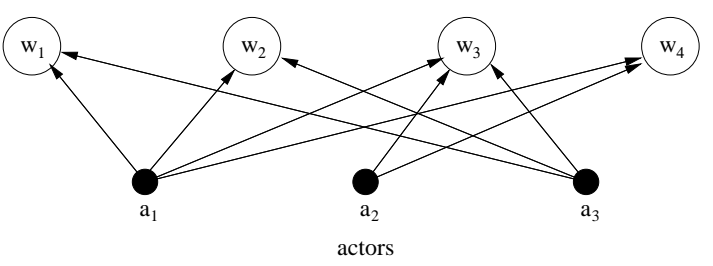

\begin{tabular}{|c|c|c|c|c|c|c|c}
$\mathrm{s}_{1}=\mathrm{a}_{1}$ & $\mathrm{~s}_{2}=\mathrm{a}_{3}$ & $\mathrm{~s}_{3}=\mathrm{a}_{1}$ & $\mathrm{~s}_{4}=\mathrm{a}_{3}$ & $\mathrm{~s}_{5}=\mathrm{a}_{2}$ & $\mathrm{~s}_{6}=\mathrm{a}_{1}$ & $\mathrm{~s}_{7}=\mathrm{a}_{2}$ & \\
\hline$\Delta \mathrm{t}_{1}$ & $\Delta \mathrm{t}_{2}$ & $\Delta \mathrm{t}_{3}$ & $\Delta \mathrm{t}_{4}$ & $\Delta \mathrm{t}_{5}$ & $\Delta \mathrm{t}_{6}$ & $\Delta \mathrm{t}_{7}$ & $\mathrm{t}$ \\
& & & & & & & \\
& & & $\mathrm{w}_{2}$ & & $\mathrm{w}_{3}$ & $\mathrm{w}_{4}$ & $\mathrm{t}$
\end{tabular}

Fig. 2. Interaction pattern extraction. The picture shows the Social Affiliation Network extracted from a speaker segmentation. The events of the network correspond to the segments $w_{j}$ and the actors are linked to the events when they talk during the corresponding segment. The actors are represented using n-tuples $\vec{x}_{a}$ where the components account for the links between actors and events.

person. The stage includes two steps: the first is the segmentation of the recordings into single speaker segments (speaker diarization), the second is the extraction of a Social Affiliation Network from the resulting speaker sequence (see upper dotted box in Figure 1).

The experiments involve two kinds of data: radio programs, where there is a single audio channel, and meeting recordings, where each participant wears a headset microphone. This requires the application of different speaker diarization techniques fully described in [23] (broadcast data) and [24] (meeting recordings). The techniques are not described here because they are not the main element of interest in this work. Section III-A shows how the output of the speaker diarization is used to build a Social Affiliation Network and represent people with n-tuples accounting for their interaction pattern.

\section{A. Affiliation Network Extraction}

The result of the speaker diarization process is that each recording is split into a sequence $S=\left\{\left(s_{i}, \Delta t_{i}\right)\right\}$, where $i \in\{1, \ldots,|S|\}, s_{i}$ is the label assigned to the speaker voice detected in the $i^{\text {th }}$ segment of audio, and $\Delta t_{i}$ is the duration of the $i^{\text {th }}$ segment. The label $s_{i}$ belongs to the set $A$ of unique speaker labels, output by the speaker diarization process (see lower part of Figure 2). The sequences extracted from the speaker diarization are used to create a Social Affiliation Network (SAN) representing the relationships between the roles. A SAN is a graph with two kinds of nodes: the actors and the events [5]. Actors can be linked to events, but no links are allowed between nodes of the same kind (see upper part of Figure 2). In the experiments, the actors correspond to the people involved in the recordings, and the events correspond to uniform non-overlapping segments spanning the whole length of the recordings. The rationale behind this choice is that actors speaking in the same interval of time are more likely to talk with one another (i.e. of interacting with one another) than actors speaking in different intervals of time. Thus, the SAN encodes information about who interacts with whom and when.

One of the main advantages of this representation is that each actor $a$ can be represented by a n-tuple $\mathbf{x}_{a}=$ $\left(x_{a 1}, \ldots, x_{a D}\right)$, where $D$ is the number of segments used as events and the component $x_{a j}$ accounts for the participation of the actor $a$ in the $j^{t h}$ event. The experiments make use of two kinds of representation. In the first one, component $x_{a j}$ is 1 if the actor $a$ talks during the $j^{\text {th }}$ segment and 0 otherwise (the corresponding n-tuples are shown at the bottom of Figure 2). In the second one, $x_{a j}$ is the number of times that actor $a$ talks during the $j^{\text {th }}$ segment. In the first case the n-tuples are binary, in the second case they have integer components higher or equal to 0 . In both cases, people that interact more with each other tend to talk during the same segments and are represented by similar n-tuples. If the roles influence the structure of the relationships between people, similar n-tuples 
should correspond to the same role.

\section{ROLE RECOGNITION}

The problem of role recognition can be formalized as follows: given a set of actors $A$ and a set of roles $\mathcal{R}$, find the function $\varphi: A \rightarrow \mathcal{R}$ mapping the actors into their actual role. In other words, the problem corresponds to finding the function $\varphi$ such that $\varphi(a)$ is the role of actor $a$.

Section III has shown that the interaction pattern of each actor $a$ is represented with a $\mathbf{n}$-tuple $\mathbf{x}_{a}=\left(x_{a 1}, \ldots, x_{a D}\right)$, where $D$ is the number of segments, that can have either binary or positive integer components. Furthermore, every actor $a$ talks for a fraction $\tau_{a}$ of the total time of the recording. Thus, each actor corresponds to a pair $\mathbf{y}_{a}=\left(\tau_{a}, \mathbf{x}_{a}\right)$.

Given a function $\varphi: A \rightarrow \mathcal{R}$ and the set of observations $Y=\left\{\mathbf{y}_{a}\right\}_{a \in A}$, the problem of assigning a role to each actor can be thought of as the maximization of the a-posteriori probability $\mathrm{p}(\varphi \mid Y)$. By applying Bayes Theorem and by taking into account that $\mathrm{p}(Y)$ is constant during recognition, this problem is equivalent to finding $\hat{\varphi}$ such that:

$$
\hat{\varphi}=\arg \max _{\varphi \in \mathcal{R}^{A}} \mathrm{p}(Y \mid \varphi) \mathrm{p}(\varphi) .
$$

where $\mathcal{R}^{A}$ is the set of all possible functions mapping actors into roles.

In order to simplify the problem, two assumptions are made: the first is that the observations are mutually conditionally independent given the roles. The second is that the observation $\mathbf{y}_{a}$ of actor $a$ only depends on its role $\varphi(a)$ and not on the role of the other actors. Equation (1) can thus be rewritten as:

$$
\hat{\varphi}=\arg \max _{\varphi \in \mathcal{R}^{A}} \mathrm{p}(\varphi) \prod_{a \in A} \mathrm{p}\left(\mathbf{y}_{a} \mid \varphi(a)\right) .
$$

The above expression is further simplified by assuming that the speaking time $\tau_{a}$ and the interaction $\mathrm{n}$-tuples $\mathbf{x}_{a}$ of actors $a$ are statistically independent given the role $\varphi(a)$, thus the last equation becomes:

$$
\hat{\varphi}=\arg \max _{\varphi \in \mathcal{R}^{A}} \mathrm{p}(\varphi) \prod_{a \in A} \mathrm{p}\left(\mathbf{x}_{a} \mid \varphi(a)\right) \mathrm{p}\left(\tau_{a} \mid \varphi(a)\right) .
$$

The probabilities appearing in the last equation have been estimated using different models to take into account the two representations of $\mathbf{x}_{a}$ described above, and to model the constraints in the distribution of roles (e.g. there must be only one anchorman in a given talk-show), i.e. to explicitly take into account the dependence between the roles.

The next sections show how $\mathrm{p}\left(\mathrm{x}_{a} \mid \varphi(a)\right), \mathrm{p}\left(\tau_{a} \mid \varphi(a)\right)$, and $\mathrm{p}(\varphi)$ are estimated in the experiments.

\section{A. Modeling Interaction Patterns}

This section shows how the probability $\mathrm{p}\left(\mathrm{x}_{a} \mid \varphi(a)\right)$ is estimated for both binary and multinomial $\mathrm{n}$-tuples $\mathbf{x}_{a}$ (see Section III-A).

When the components of the n-tuple $\mathbf{x}_{a}$ are binary, i.e. $x_{a j}=1$ when actor $a$ talks during segment $j$ and 0 otherwise, the most natural way of modeling $\mathbf{x}_{a}$ is to use independent Bernoulli discrete distributions:

$$
\mathrm{p}(\mathbf{x} \mid \mu)=\prod_{j=1}^{D} \mu_{j}^{x_{j}}\left(1-\mu_{j}\right)^{1-x_{j}},
$$

where $D$ is the number of events in the network (see Section III), and $\mu=\left(\mu_{1}, \ldots, \mu_{D}\right)$ is the parameter vector of the distribution. A different Bernoulli distribution is trained for each role. The maximum likelihood estimates of the parameters $\mu_{r}$ for a given role $r$ are as follows [6]:

$$
\mu_{r j}=\frac{1}{\left|A_{r}\right|} \sum_{a \in A_{r}} x_{a j},
$$

where $A_{r}$ is the set of actors playing the role $r$ in the training set, and $\mathbf{x}_{a}$ is the n-tuple representing the actor $a$.

When the components $\mathbf{x}_{j}$ correspond to the number of times that actor $a$ talks during event $j$, i.e. when the components are integers greater or equal to 0 , they can be represented with a vector $\mathbf{z}_{i}=\left(z_{i 1}, \ldots, z_{i T}\right)$ where $T$ is the maximum number of times that an actor can talk during a given event, $z_{i j} \in\{0,1\}$, and $\sum_{j=1}^{T} z_{i j}=1$ (one-out-of- $K$ ). In other words, $x_{i}$ is represented with a $T$-dimensional vector where all the components are 0 except one, i.e. the component $z_{i n}=1$, where $n$ is the number of times that the actor represented by $\mathbf{x}$ talks during event $i$. As a result, $\mathbf{x}$ is represented as a $\mathrm{n}$-tuple of vectors $\mathbf{z}=\left(\mathbf{z}_{1}, \ldots, \mathbf{z}_{D}\right)$ and can be modeled as a product of independent Multinomial distributions:

$$
\mathrm{p}(\mathbf{z} \mid \mu)=\prod_{i=1}^{D} \prod_{j=1}^{T} \mu_{i j}^{z_{i j}} .
$$

The parameters $\mu$ can be estimated by maximizing the likelihood of $\mathrm{p}(\mathbf{z} \mid \mu)$ over a training set $\mathcal{X}$. This leads to a closed form expression for the parameters:

$$
\mu_{i j}=\frac{1}{\left|A_{r}\right|} \sum_{a \in A_{r}} z_{a i j} .
$$

\section{B. Modeling Durations}

Given a labeled training set, there is a set $A_{r}$ of actors playing role $r, \mathrm{p}(\tau \mid r)$ is estimated using a Gaussian Distribution $\mathcal{N}\left(\tau \mid \mu_{r}, \sigma_{r}\right)$, where $\mu_{r}$ and $\sigma_{r}$ are the sample mean and variance respectively:

$$
\begin{gathered}
\mu_{r}=\frac{1}{\left|A_{r}\right|} \sum_{a \in A_{r}} \tau_{a}, \\
\sigma_{r}=\frac{1}{\left|A_{r}\right|} \sum_{a \in A_{r}}\left(\tau_{a}-\mu_{r}\right)^{2} .
\end{gathered}
$$

This corresponds to a Maximum Likelihood estimate, where a different Gaussian distribution is obtained for each role.

\section{Estimating Role Probabilities}

This subsection shows how the a-priori probability $\mathrm{p}(\varphi(a))$ of actor $a$ playing role $\varphi(a)$ is estimated. Two approaches are proposed: the first is based on the assumption that roles are independent and does not take into account the constraints that 
the role distribution across different participants in a given recording must respect, e.g. there is only one Anchorman in a talk-show, there is only one Project Manager in a meeting, etc. The second approach considers the roles to be dependent and takes into account the above constraints.

1) Modeling Independent Roles: The first approach assumes that the roles are independent and thus that $\mathrm{p}(\varphi)$ is simply the product of the a-priori probabilities of the roles assigned through $\varphi$ to the different actors:

$$
\mathrm{p}(\varphi)=\prod_{a \in A} \mathrm{p}(\varphi(a))
$$

The a-priori probability of observing the role $r$ can be estimated as follows:

$$
\mathrm{p}(\varphi(a))=\frac{N_{\varphi(a)}}{N}
$$

where $N$ and $N_{\varphi(a)}$ are the total number of actors and the total number of actors playing role $\varphi(a)$ in the training set.

Using the above approach, Equation (2) boils down to

$$
\hat{\varphi}=\arg \max _{\varphi \in \mathcal{R}^{A}} \prod_{a \in A} \mathrm{p}\left(\mathbf{x}_{a} \mid \varphi(a)\right) \mathrm{p}\left(\tau_{a} \mid \varphi(a)\right) \mathrm{p}(\varphi(a)) .
$$

and the role recognition process simply consists in assigning each actor the role $\varphi(a)$ that maximizes the probability $\mathrm{p}\left(\mathbf{x}_{a} \mid \varphi(a)\right) \mathrm{p}\left(\tau_{a} \mid \varphi(a)\right) \mathrm{p}(\varphi(a))$.

2) Modeling Dependent Roles: The second approach tries to model the constraints that the role distribution of a given recording must respect. For example, there must be only one Anchorman in a talk show while the number of Guests can change at each edition of the talk show. In this case, the roles played by the different recording participants cannot be considered independent, and $\mathrm{p}(\varphi)$ cannot be written as the product of the a-priori probabilities of the roles (like in Equation 10).

A given mapping $\varphi \in \mathcal{R}^{A}$ corresponds to a distribution of roles across the different recording participants where each role is played by a certain number of actors. The constraints to be respected are expressed in terms of the number of actors that can play a given role (e.g., only one actor can be the Anchorman). Thus, $\mathrm{p}(\varphi)$ must be different from 0 only for those distributions of roles that respect the constraints. The number of possible actors playing some roles is actually predetermined (i.e. exactly $n_{r}$ actors must play role $r$ ), while for others the only available a-priori information is that at least one person must play the role (i.e. $n_{r}>0$ ).

According to the above, $\mathrm{p}(\varphi)$ is modeled with a product of Multinomial distributions [6]:

$$
\mathrm{p}(\varphi)=\prod_{r \in \mathcal{R}} \mathrm{p}\left(\mathbf{z}_{r} \mid \mu_{r}\right)
$$

where $\mathbf{z}_{r}$ is a one-out-of- $K$ (see Section IV-A) representation of the number of times a role can be played in a given recording, and $\mu_{r}$ is the parameter vector.

We can divide the set $\mathcal{R}^{A}$ in classes $\left\{C_{g}\right\}$ where all mappings lead to a role distribution where the same role is played always the same number of times. We assume that all mappings $\varphi$ in the same class have the same probability. Thus, the probability of observing a given assignment is:

$$
\mathrm{p}(\varphi)=\frac{\prod_{r \in \mathcal{R}} \mathrm{p}\left(\mathbf{z}_{r} \mid \mu_{r}\right)}{\left|C_{g}\right|}
$$

Then in the second model, Equation (2) can be rewritten as:

$$
\hat{\varphi}=\arg \max _{\varphi \in \mathcal{R}^{A}} \mathrm{p}(\varphi) \prod_{a \in A} \mathrm{p}\left(\mathbf{x}_{a} \mid \varphi(a)\right) \mathrm{p}\left(\tau_{a} \mid \varphi(a)\right) .
$$

where $\mathrm{p}(\varphi)$ is the expression of Equation 14. Maximizing this product using a brute-force approach is not tractable if the number of actors is high. Therefore, we used simulated annealing [25] to approximate the best mapping for each recording.

\section{EXPERIMENTS AND RESULTS}

The next four sections describe data and roles, performance measures, experimental setup and role recognition results.

\section{A. Data and Roles}

The experiments of this work have been performed over three different corpora referred to as $\mathrm{C} 1, \mathrm{C} 2$ and $\mathrm{C} 3$ in the following. $\mathrm{C} 1$ contains all news bulletins (96 in total) broadcasted by Radio Suisse Romande (the French speaking Swiss National broadcasting service) during February 2005. The average length of $\mathrm{C} 1$ recordings is 11 minutes and 50 seconds, and the average number of participants is 12 . C2 contains all talk-shows (27 in total) broadcasted by Radio Suisse Romande during February 2005. All C2 recordings are one hour long and the average number of participants is 25 . C3 is the AMI meeting corpus [7], a collection of 138 meeting recordings involving 4 persons each and with an average length of 19 minutes and 50 seconds. While $\mathrm{C} 1$ and $\mathrm{C} 2$ contain real-world news and talk-shows, the meetings in $\mathrm{C} 3$ are a simulation and the participants act roles they do not play in their real life.

The roles of $\mathrm{C} 1$ and $\mathrm{C} 2$ share the same names and correspond to similar functions: the Anchorman (AM), i.e. the person managing the program, the Second Anchorman (SA), i.e. the person supporting the AM, the Guest (GT), i.e. the person invited to report about a single and specific issue, the Interview Participant (IP), i.e. interviewees and interviewers, the Headline Reader (HR), i.e. the speaker reading a short abstract at the beginning of the program, and the Weather Man (WM), i.e. the person reading the weather forecasts. However, even if the roles have the same name and correspond to roughly the same functions, they are played in a different way in $\mathrm{C} 1$ and $\mathrm{C} 2$ (e.g., consider how different is the behavior of an anchorman in news supposed to inform and in talkshows supposed to entertain). In C3, the role set is different and contains the Project Manager (PM), the Marketing Expert (ME), the User Interface Expert (UI), and the Industrial Designer (ID). See Table II for the distribution of roles in the corpora. 
TABLE II

Role Distribution. THE TABle Reports the PERCENTAGE Of TIME EACH ROLE ACCOUNTS FOR IN C1, C2 AND C3.

\begin{tabular}{|c|c|c|c|c|c|c||c|c|c|c|}
\hline Corpus & AM & SA & GT & IP & HR & WM & PM & ME & UI & ID \\
\hline C 1 & $41.2 \%$ & $5.5 \%$ & $34.8 \%$ & $4.0 \%$ & $7.1 \%$ & $6.3 \%$ & N/A & N/A & N/A & N/A \\
\hline C 2 & $17.3 \%$ & $10.3 \%$ & $64.9 \%$ & $0.0 \%$ & $4.0 \%$ & $1.7 \%$ & N/A & N/A & N/A & N/A \\
\hline C 3 & N/A & N/A & N/A & N/A & N/A & N/A & $36.6 \%$ & $22.1 \%$ & $19.8 \%$ & $21.5 \%$ \\
\hline
\end{tabular}

\section{B. Speaker Diarization Results}

The interaction patterns used at the role recognition step are extracted from the speaker segmentation obtained with the two different diarization processes (see Sections III). Errors in the diarization (e.g. people detected as speaking when they are silent, or multiple voices attributed to a single speaker) lead to spurious interactions that can mislead the role recognition process.

The effectiveness of the diarization is measured with the Purity $\pi$, a metric showing on one hand to what extent all feature vectors corresponding to a given speaker are detected as belonging to the same voice, and on the other hand to what extent all vectors detected as a single voice actually correspond to a single speaker. The Purity ranges between 0 and 1 (the higher the better) and it is the geometric mean of two terms: the average cluster purity $\pi_{c}$ and the average speaker purity $\pi_{s}$. The definition of $\pi_{c}$ is as follows:

$$
\pi_{c}=\sum_{k=1}^{N_{c}} \sum_{l=1}^{N_{s}} \frac{n_{k}}{N} \frac{n_{l k}^{2}}{n_{k}^{2}}
$$

where $N$ is the total number of feature vectors, $N_{s}$ is the number of speakers, $N_{c}$ is the number of voices detected in the diarization process, $n_{l k}$ is the number of vectors belonging to speaker $l$ that have been attributed to voice $k$, and $n_{k}$ is the number of feature vectors in voice $k$. The definition of $\pi_{s}$ is as follows:

$$
\pi_{s}=\sum_{l=1}^{N_{s}} \sum_{k=1}^{N_{c}} \frac{n_{l}}{N} \frac{n_{l k}^{2}}{n_{l}^{2}}
$$

(see above for the meaning of the symbols).

The application of the speaker diarization process in the case of radio programs requires the setting of the initial number of states $M$ in the fully connected Hidden Markov Model (see Section III). The value of $M$ must be significantly higher than the number of expected speakers for the diarization process to work correctly. In our experiments, we set a-priori $M=30$ for $\mathrm{C} 1$ and $M=90$ for $\mathrm{C} 2$. No other values have been tested. The average purity is 0.81 for $\mathrm{C} 1$ and 0.79 for $\mathrm{C} 2$. The average purity for $\mathrm{C} 3$ is 0.99 . The difference in purity is explained by the different experimental conditions and methods used to obtain the speaker segmentation.

\section{Experimental Setup}

The experiments are based on a $K$-fold cross-validation approach [6]. The corpora are split into $K$ equally sized parts of which $K-1$ are used as training set, while the remaining one is used as the test set. Each of the $K$ parts is used iteratively as the test set so that the experiments can be performed over the whole dataset while still preserving a rigorous separation between training and test set. In the case of our experiments, $K=5$ and each subset contains $20 \%$ of the data. The only hyperparameter to be set is the number $D$ of segments used as events in the Social Affiliation Network. At each iteration of the $K$-fold cross-validation, $\mathrm{D}$ is varied such that the value giving the highest role recognition results over the training set has been retained for testing. In this way, a rigorous separation between the training and test set has been observed for the setting of the hyperparameter as well.

The statistical significance of performance differences is assessed with the Kolmogorov-Smirnov test [26]. The advantage of this test is that it does not make assumptions about the distribution of the performance (unlike the $t$-test that assumes the performances following a Gaussian distribution) and it is adapted to continuous distributions (unlike the $\chi^{2}$ test that requires the distributions to be made discrete through histogramming).

\section{Role Recognition Results}

Table III reports the results achieved over $\mathrm{C} 1$ and $\mathrm{C} 2$, Table IV those obtained for $\mathrm{C} 3$. The performance is measured in terms of accuracy, i.e. the percentage of time correctly labeled in terms of role in the test set. Each accuracy value is accompanied by the standard deviation of the accuracies achieved over the different recordings of each corpus. The distribution used to model the interaction patterns is indicated with $B$ (Bernoulli) and $M$ (Multinomial). The approach used to estimate the a-priori role probabilities is indicated with $I$ (independence) and $D$ (dependence).

Modeling the dependence between roles leads to statistically significant improvements for $\mathrm{C} 2$ and $\mathrm{C} 3$, while it decreases the performance for $\mathrm{Cl}$. One probable explanation is that $\mathrm{C} 1$ presents more variability in the number of people playing a given role, thus $\mathrm{p}(\varphi)$ (see Section IV-C) cannot be estimated as reliably as for the other corpora. However, these results suggest that taking into account the dependence across roles is beneficial as long as $\mathrm{p}(\varphi)$ can be estimated reliably. To the best of our knowledge, this is the first attempt to model explicitly the dependence between roles and the results provide a first assessment of what can be expected, at least for the approach proposed here, in terms of performance improvement.

For the three corpora, the differences between the performances achieved using Bernoulli and Multinomial distributions are not statistically significant. This suggests that the important information is presence/absence (conveyed by the Bernoulli distribution) and not number of times a speaker talks during an event (conveyed by the Multinomial). This is not surprising because the most important aspect encoded by Social Affiliation Networks (at least for the approach proposed 
TABLE III

ROLE RECOGNITION PERFORMANCE FOR C1 AND C2. THE TABLE REPORTS BOTH THE OVERALL ACCURACY AND THE ACCURACY FOR EACH ROLE. "B" STANDS FOR Bernoulli, "M" STANDS FOR Multinomial, "I"

STANDS FOR ROLES Independence, AND "D" STANDS FOR ROLES

dependence. THE OVERALL ACCURACY IS ACCOMPANIED BY THE STANDARD DEVIATION $\sigma$ OF THE PERFORMANCES ACHIEVED OVER THE SINGLE RECORDINGS. THE UPPER PART OF THE TABLE REPORTS THE RESULTS OBTAINED OVER THE OUTPUT OF THE SPEAKER SEGMENTATION, THE LOWER PART REPORTS THE RESULTS OBTAINED OVER THE MANUAL SPEAKER SEGMENTATION.

\begin{tabular}{|c|c|c|c|c|c|c|c|}
\hline & all $(\sigma)$ & $\mathrm{AM}$ & SA & GT & IP & HR & WM \\
\hline \multicolumn{8}{|c|}{ Automatic Speaker Segmentation } \\
\hline C1 $(\mathrm{B}, \mathrm{I})$ & $81.7(6.9)$ & 98.0 & 4.0 & 92.0 & 5.6 & 55.9 & 76.8 \\
\hline $\mathrm{C} 1(\mathrm{~B}, \mathrm{D})$ & $62.7(16.5)$ & 89.9 & 4.2 & 68.9 & 9.0 & 11.0 & 10.1 \\
\hline C1 $(\mathrm{M}, \mathrm{I})$ & $82.4(7.1)$ & 97.8 & 4.8 & 92.2 & 4.2 & 64.3 & 78.2 \\
\hline C1 (M,D) & $62.3(16.7)$ & 88.7 & 3.4 & 70.2 & 4.5 & 7.0 & 15.4 \\
\hline $\mathrm{C} 2(\mathrm{~B}, \mathrm{I})$ & $83.2(6.7)$ & 75.0 & 88.3 & 91.5 & N/A & 29.1 & 9.0 \\
\hline $\mathrm{C} 2(\mathrm{~B}, \mathrm{D})$ & $87.5(4.4)$ & 77.1 & 92.1 & 93.2 & N/A & 91.0 & 17.7 \\
\hline $\mathrm{C} 2(\mathrm{M}, \mathrm{I})$ & $84.0(6.5)$ & 68.7 & 92.2 & 89.7 & N/A & 83.7 & 15.4 \\
\hline $\mathrm{C} 2(\mathrm{M}, \mathrm{D})$ & $87.8(4.3)$ & 77.1 & 92.1 & 93.2 & N/A & 98.4 & 16.3 \\
\hline \multicolumn{8}{|c|}{ Manual Speaker Segmentation } \\
\hline $\mathrm{C} 1(\mathrm{~B}, \mathrm{I})$ & $95.1(4.6)$ & 100 & 88.5 & 98.3 & 13.9 & 100 & 97.9 \\
\hline $\mathrm{C} 1(\mathrm{~B}, \mathrm{D})$ & $66.7(12.5)$ & 96.9 & 5.2 & 66.9 & 11.8 & 21.9 & 12.5 \\
\hline C1 $(\mathrm{M}, \mathrm{I})$ & $97.0(4.2)$ & 100 & 86.5 & 98.7 & 61.5 & 100 & 97.9 \\
\hline $\mathrm{C} 1(\mathrm{M}, \mathrm{D})$ & $67.5(9.6)$ & 99.0 & 6.2 & 72.0 & 3.3 & 6.2 & 10.4 \\
\hline C2 $(\mathrm{B}, \mathrm{I})$ & $96.2(2.6)$ & 96.3 & 100 & 96.6 & N/A & 100 & 70.4 \\
\hline $\mathrm{C} 2(\mathrm{~B}, \mathrm{D})$ & $96.1(5.8)$ & 96.3 & 96.3 & 97.7 & N/A & 100 & 33.3 \\
\hline $\mathrm{C} 2(\mathrm{M}, \mathrm{I})$ & $95.8(7.7)$ & 96.3 & 96.3 & 95.7 & N/A & 100 & 81.5 \\
\hline C2 (M,D) & $98.1(2.1)$ & 100 & 100 & 98.6 & N/A & 100 & 48.1 \\
\hline
\end{tabular}

TABLE IV

ROLE RECOGNITION PERFORMANCE FOR C3. THE TABLE REPORTS BOTH THE OVERALL ACCURACY AND THE ACCURACY FOR EACH ROLE. "B" STANDS FOR Bernoulli, "M" STANDS FOR Multinomial, "I" STANDS FOR ROLES Independence, AND "D” STANDS FOR ROLES dependence. THE OVERALL ACCURACY IS ACCOMPANIED BY THE STANDARD DEVIATION $\sigma$ OF THE PERFORMANCES ACHIEVED OVER THE SINGLE RECORDINGS. THE UPPER PART OF THE TABLE REPORTS THE RESULTS OBTAINED OVER THE OUTPUT OF THE SPEAKER SEGMENTATION, THE LOWER PART REPORTS THE RESULTS OBTAINED OVER THE MANUAL SPEAKER SEGMENTATION.

\begin{tabular}{|c|c|c|c|c|c|}
\hline & all $(\sigma)$ & PM & ME & UI & ID \\
\hline \multicolumn{6}{|c|}{ Automatic Speaker Segmentation } \\
\hline C3 (B,I) & $46.0(24.7)$ & 79.6 & 13.1 & 41.4 & 20.3 \\
\hline $\mathrm{C} 3(\mathrm{~B}, \mathrm{D})$ & $46.4(30.0)$ & 68.7 & 26.0 & 32.9 & 25.7 \\
\hline C3 $(\mathrm{M}, \mathrm{I})$ & $39.3(24.9)$ & 67.4 & 18.0 & 19.3 & 25.6 \\
\hline C3 (M,D) & $43.7(31.3)$ & 67.4 & 28.7 & 22.0 & 24.3 \\
\hline \multicolumn{6}{|c|}{ Manual Speaker Segmentation } \\
\hline C3 (B,I) & $51.2(24.2)$ & 83.3 & 15.9 & 42.0 & 29.0 \\
\hline $\mathrm{C} 3(\mathrm{~B}, \mathrm{D})$ & $56.0(33.0)$ & 76.1 & 37.7 & 40.6 & 41.3 \\
\hline C3 (M,I) & $43.7(27.3)$ & 67.4 & 17.4 & 39.1 & 21.7 \\
\hline C3 (M,D) & $52.6(27.6)$ & 76.8 & 29.0 & 34.1 & 33.3 \\
\hline
\end{tabular}

in this work) is who interacts with whom and not how much someone interacts with someone else.

Overall, roles in meeting data appear to be harder to model for several reasons. On one hand, roles in meeting are informal, i.e. they correspond to a position in a given social system and do not correspond to stable behavioral patterns like in the case of the formal roles in broadcast data. On the other hand, the meetings in $\mathrm{C} 3$ are not real-world, i.e. the participants act in a scenario that does not correspond to their real lives. Not surprisingly, the meeting role recognized with highest accuracy is the Project Manager (PM). In fact, the PM plays also the role of chairman, i.e. a formal role that influences the actual interaction pattern of the people that play it. The performance difference when passing from manual (ground truth) to automatic speaker diarization is statistically significant for C1 and C2 (see Tables III and IV). The difference is not significant for C3 because the purity of the speaker segmentation for such a corpus is 0.99, i.e. it corresponds almost perfectly to the groundtruth speaker segmentation. In contrast, the difference is significant for $\mathrm{C} 1$ and $\mathrm{C} 2$ because in this case the speaker diarization process produces more errors and the purity is around 0.8 , i.e. the output of the speaker diarization is significantly different from the groundtruth speaker segmentation. The difference in accuracy is around 10 percent (statistically significant) and this is mostly due to the small differences ( 2 seconds on average) between the actual speaker changes and the changes as detected by the diarization process. The sum of all the misalignments, on average, to roughly 10 percent of the recording length and this is the probable explanation of the performance difference when passing from manual to automatic speaker segmentations.

The rest of the errors are due to limits of the role recognition approach that cannot distinguish between different roles when the associated interaction patterns are too similar. This is true for example, in the case of the low performance of the IP in corpus $\mathrm{C} 1$. The interaction pattern of the IP role is similar to that of the Guest, but the latter has higher a-priori probability, so it is usually favored as the output of the recognizer.

A qualitative comparison with other approaches is possible only for some works which use parts of the same data as ours. Both [21][22] perform experiments over a subset of the AMI meeting corpus (around 5 hours of material). The performance in [21] is around $80 \%$, almost twice as much as our approach over the same data (see Section V). However, as the goal is to detect the two most dominant persons, the probability of assigning each person the correct role is $50 \%$, while it is only $25 \%$ in our case. The work in [22] reports a $65 \%$ recognition rate of the Project Manager, while our work achieves, over the same role, an accuracy of $79 \%$. Considering that our experiments are performed over the whole AMI meeting corpus, while the experiments of [21][22] take into account only a subset of 5 hours, our approach seems to be more effective in both cases, though the task is not the same. The work in [19] uses the whole AMI corpus, but it applies a different experimental setup. However it performs exactly the same task as this work and the role recognition rate is around $60 \%$.

\section{CONCLUSiOnS AND Future Work}

This paper has presented an approach for the automatic recognition of roles in multiparty recordings. The problem of role recognition has been addressed only recently in the literature, but it attracts an increasingly growing interest because is a key point in the automatic analysis of social interactions [1][2]. The proposed approach has been tested over roughly 90 hours of material, one of the biggest datasets ever used in the literature for this task. To the best of our knowledge, this is the first work that compares the performance of an approach over both informal and formal roles (See Section II for the 
difference between the two types of role), showing how the role typology influences the effectiveness of the recognition.

The results show that the recognition accuracy is higher than $85 \%$ in the case of broadcast data, and it is around $45 \%$ in the case of meeting recordings. There are several possible reasons for such a difference. The first, and probably most important, is that broadcast data include formal roles, while meetings include informal ones. Formal roles are easier to model because they impose constraints on the behavior of people that can be detected, represented and modeled with probabilistic approaches (like in the case of this work). In contrast, informal roles do not necessarily constrain behavior and so automatic recognition is more difficult through approaches like the one presented in this work, at least for the aspect of behavior used as role evidence in this work, i.e. who talks with whom and when.

The second is that the broadcast data is real, while the meeting data is acted. The meetings do not involve people playing the role they actually have in their life, but volunteers that simulate an artificially assigned role they have never played before. This is likely to reduce significantly the performance of any role recognition method.

In the case of the broadcast data, the performance is sufficient to browse effectively the data (users can quickly find segments corresponding to a given role and the mismatch between the ground truth and the automatic output rarely exceeds a few seconds). In the case of meeting recordings, the approach is effective only to identify the Project Manager. This allows one to effectively follow the progress of the meeting because the PM plays the chairman role as well and, as such, is responsible for following the agenda through her/his interventions.

The main limitation of the current approach is that it does not take into account any sequential information. The role of the person speaking at turn $n$ is likely to have a statistical influence on the role of the person speaking at turn $n+1$. This kind of information could be modeled using probabilistic sequence models (e.g. Hidden Markov Models), as well as statistical language models (e.g., $N$-grams). Furthermore, the approach proposed in this work uses only the co-occurence turn-taking patterns as role evidence, while other behavioral cues can be extracted from both audio (e.g., prosodic features), and video (e.g., gestures). Both above limitations will be the subject of future work.

\section{ACKNOWLEDGMENTS}

The authors wish to thank John Dines and François Fleuret for technical support, as well as Hayley Hung and Alfred Dielmann for commenting on the draft.

\section{REFERENCES}

[1] A. Vinciarelli, M. Pantic, and H. Bourlard, "Social Signal Processing: Survey of an emerging domain," Image and Vision Computing, to appear, 2009.

[2] A. Vinciarelli, M. Pantic, H. Bourlard, and A. Pentland, "Social Signal Processing: State-of-the-art and future perspectives of an emerging domain," in Proceedings of the ACM International Conference on Multimedia, 2008, pp. 1061-1070.
[3] D. Gatica-Perez, "Automatic nonverbal analysis of social interaction in small groups: a review," Image and Vision Computing, to appear, 2009.

[4] H. Tischler, Introduction to Sociology. Harcourt Brace College Publishers, 1990.

[5] S. Wasserman and K. Faust, Social Network Analysis. Cambridge University Press, 1994.

[6] C. Bishop, Pattern Recognition and Machine Learning. Springer Verlag, 2006.

[7] I. McCowan, J. Carletta, W. Kraaij, S. Ashby, S. Bourban, M. Flynn, M. Guillemot, T. Hain, J. Kadlec, V. Karaiskos, M. Kronenthal, G. Lathoud, M. Lincoln, A. Lisowska, W. Post, D. Reidsma, and P. Wellner, "The AMI meeting corpus," in Proceedings of the 5th International Conference on Methods and Techniques in Behavioral Research, 2005, p. 4.

[8] Y. Liu, "Initial study on automatic identification of speaker role in broadcast news speech," in Proceedings of the Human Language Technology Conference of the NAACL, Companion Volume: Short Papers, June 2006, pp. 81-84.

[9] S. Maskey and J. Hirschberg, "Automatic summarization of broadcast news using structural features," in Proceedings of the European Conference on Speech Communication and Technology, 2003, pp. 1173-1176.

[10] A. Vinciarelli, "Sociometry based multiparty audio recordings summarization," in Proceedings of the International Conference on Pattern Recognition, 2006, pp. 1154-1157.

[11] A. Vinciarelli and S. Favre, "Broadcast news story segmentation using Social Network Analysis and Hidden Markov Models," in Proceedings of the ACM International Conference on Multimedia, 2007, pp. 261-264.

[12] C. Weng, W. Chu, and J. Wu, "Movie analysis based on roles social network," in Proceedings of IEEE International Conference on Multimedia and Expo, 2007, pp. 1403-1406.

[13] A. Vinciarelli, "Speakers role recognition in multiparty audio recordings using Social Network Analysis and duration distribution modeling," IEEE Transactions on Multimedia, vol. 9, no. 6, pp. 1215-1226, 2007.

[14] J. Levine and R. Moreland, "Small groups," in The handbook of social psychology, D. Gilbert and G. Lindzey, Eds. Oxford University Press, 1998, vol. 2, pp. 415-469.

[15] R. Barzilay, M. Collins, J. Hirschberg, and S. Whittaker, "The rules behind the roles: identifying speaker roles in radio broadcasts," in Proceedings of the $17^{\text {th }}$ National Conference on Artificial Intelligence, 2000, pp. 679-684.

[16] C. Weng, W. Chu, and J. Wu, "Rolenet: Movie analysis from the perspective of social networks," IEEE Transactions on Multimedia, vol. 11, no. 2, pp. 256-271, 2009.

[17] F. Pianesi, M. Zancanaro, E. Not, C. Leonardi, V. Falcon, and B. Lepri, "A multimodal annotated corpus of consensus decision making meetings," The Journal of Language Resources and Evaluation, vol. 41, no. 3-4, pp. 409-429, 2008.

[18] S. Banerjee and A. Rudnicky, "Using simple speech based features to detect the state of a meeting and the roles of the meeting participants,' in Proceedings of International Conference on Spoken Language Processing, no. 2-3, 2004, pp. 221-231.

[19] K. Laskowski, M. Ostendorf, and T. Schultz, "Modeling vocal interaction for text-independent participant characterization in multi-party conversation," in In proceedings of the 9th ISCA/ACL SIGdial Workshop on Discourse and Dialogue, June 2008, pp. 148-155.

[20] N. Garg, S. Favre, H. Salamin, D. Hakkani-Tür, and A. Vinciarelli, "Role recognition for meeting participants: an approach based on lexical information and Social Network Analysis," in Proceedings of the ACM International Conference on Multimedia, 2008, pp. 693-696.

[21] D. Jayagopi, H. Hung, C. Yeo, and D. Gatica-Perez, "Predicting the dominant clique in meetings through fusion of nonverbal cues," in Proceedings of the ACM International Conference on Multimedia, 2008, pp. 809-812.

[22] D. Jayagopi, S. Ba, J. Odobez, and D. Gatica-Perez, "Predicting two facets of social verticality in meetings from five-minute time slices and nonverbal cues," in Proceedings of the International Conference on Multimodal Interfaces, 2008, pp. 45-52.

[23] J. Ajmera, "Robust audio segmentation," Ph.D. dissertation, École Polytechnique Fédérale de Lausanne (EPFL), 2004.

[24] J. Dines, J. Vepa, and T. Hain, "The segmentation of multi-channel meeting recordings for automatic speech recognition," in Proceedings of Interspeech, 2006, pp. 1213-1216.

[25] S. Kirkpatrick, C. D. Gelatt, and M. P. Vecchi, "Optimization by simulated annealing," Science, vol. 220, pp. 671-680, 1983.

[26] F. Massey Jr., "The Kolmogorov-Smirnov test for goodness of fit," Journal of the American Statistical Association, pp. 68-78, 1951. 